\title{
ANÁLISE DAS NOVAS DIRETRIZES CURRICULARES NACIONAIS COM APLICAÇÃO EM UM CURSO DE ENGENHARIA MECÂNICA
}

EdsonBaal-ed.baal@gmail.com

Instituto Federal de Educação, Ciência e Tecnologia do Rio Grande do Sul, Campus Ibirubá Rua Nelsi Ribas Fritsch, 1111

98200-000 - Ibirubá - Rio Grande do Sul

Resumo: Este trabalho apresenta uma revisão das novas Diretrizes Curriculares Nacionais do Curso de Graduação em Engenharia (DCNs) e apresenta ações em conformidade com tais diretrizes e que já são realizadas no âmbito do curso de Engenharia Mecânica do Instituto Federal de Educação, Ciência e Tecnologia do Rio Grande do Sul (IFRS) Campus Ibirubá. As novas DCNs foram publicadas no ano de 2019, com o intuito de aprimorar a qualidade dos cursos de graduação em Engenharia no país, visto as demandas dos setores da sociedade onde atuam os Engenheiros. A adequação às diretrizes leva a uma importante reflexão sobre o projeto pedagógico do curso, sendo evidenciado nesse processo pontos positivos e negativos do curso de graduação. Na revisão do documento das novas DCNs destacam-se o incentivo a utilização de metodologias ativas e o estímulo à iniciação científica dos estudantes. A busca por algumas ações já realizadas no âmbito do curso ocorreu através da pesquisa por trabalhos publicados pelos estudantes em eventos científicos institucionais. Foram elencados vinte trabalhos que demonstram pontos em comum entre o curso e o que prima as novas DCNs, no que tange as metodologias ativas e o incentivo à iniciação científica dos estudantes. Ainda, há a necessidade de sistematizar e formalizar tais ações no projeto Pedagógico do curso para a devida adequação às novas DCNs.

Palavras-chave: Diretrizes curriculares nacionais. Engenharia mecânica. Metodologias ativas.

\section{INTRODUÇÃO}

Este trabalho busca apresentar ações desenvolvidas no âmbito do curso de Engenharia Mecânica do Instituto Federal de Educação, Ciência e Tecnologia do Rio Grande do Sul (IFRS), Campus Ibirubá, e que vão ao encontro das novas Diretrizes Curriculares Nacionais do Curso de Graduação em Engenharia (DCNs).

Essa análise é realizada em um momento de reflexão e reformulação dos cursos de graduação em Engenharia. Para isso, realiza-se uma revisão do documento das novas DCNs, com o destaque no que diz respeito à metodologias ativas, e ao incentivo à iniciação científica dos estudantes. Na sequência, busca-se por trabalhos publicados pelos estudantes em eventos científicos institucionais, que evidenciam a afinidade do curso com metodologias ativas e incentivo à iniciação científica dos estudantes.

As DCNs foram publicas pelo Conselho Nacional de Educação no ano de 2019, fixando o prazo de três anos para a implementação das diretrizes pelos cursos de engenharia. Portanto atualmente, trabalhos de reformulação de Projeto Pedagógico de Curso (PPC) estão em franca 
expansão, o que leva a uma reflexão sobre o contexto geral dos cursos de engenharia nas instituições de ensino.

De acordo com a Mobilização Empresarial pela Inovação (2020), as novas diretrizes tem como objetivos elevar a qualidade dos cursos, onde os conteúdos são resultados das competências estabelecidas como metas para a formação de engenheiros capazes de empreender e inovar nos diversos campos de atuação da engenharia. Busca-se o engajamento do aluno através de seu protagonismo na aprendizagem, aproximação com práticas e problemas reais da sociedade.

Assim, no Capítulo 2, apresenta-se os principais pontos das novas DCNs. No Capítulo 3 apresenta-se algumas ações que vêm ao encontro das novas DCNs realizadas no curso de Engenharia Mecânica do IFRS Campus Ibirubá. Por fim, no Capítulo 4 tem-se as considerações finais e as referências.

\section{AS NOVAS DIRETRIZES CURRICULARES NACIONAIS DO CURSO DE GRADUAÇÃO EM ENGENHARIA}

\subsection{Perfil do egresso}

Conforme Brasil (2019), o PPC de engenharia necessita estabelecer o perfil do egresso no contexto em que a instituição de ensino está inserida, levando em conta, além das demandas locais, as demandas nacionais e mundiais em uma perspectiva globalizada da atuação em engenharia.

Conforme a Comissão Nacional para Implantação das Novas Diretrizes Curriculares Nacionais do Curso de Graduação em Engenharia (CN-DCNs) (2020), a tratativa sobre competências na elaboração do projeto pedagógico do curso diferencia-se da abordagem tradicional, onde inicialmente definia-se os conteúdos a serem trabalhados ao longo do curso. Agora, parte-se das competências necessárias delineadas no perfil do egresso.

Assim, para delinear as competências do perfil do egresso, as DCNs apresentam princípios a serem considerados na elaboração do PPC, conforme Brasil (2019, p. 25), são eles:

I. Formular e conceber soluções desejáveis de Engenharia, analisando e compreendendo a necessidade dos usuários e seu contexto;

II. Analisar e compreender os fenômenos físicos e químicos por meio de modelos simbólicos, físicos e outros, uma vez verificados e validados por experimentação;

III. Conceber, projetar e analisar sistemas, produtos (bens e serviços), componentes ou processos;

IV. Implantar, supervisionar e controlar as soluções de Engenharia;

V. Comunicar-se eficazmente nas formas escrita, oral e gráfica;

VI. Trabalhar e liderar equipes multidisciplinares;

VII. Conhecer e aplicar com ética a legislação e os atos normativos no âmbito do exercício da profissão;

Tais princípios colaboram na construção de um perfil de egresso generalista, o qual ainda, deve ser contemplado com as particularidades da habilitação ou ênfase do curso. Também são explicitados princípios para perfil do egresso, com atuação em diferentes campos da engenharia, conforme Brasil (2019, p. 26):

I. Em todo o ciclo de vida e contexto do projeto de produtos (bens e serviços) e de seus componentes, sistemas e processos produtivos, inclusive inovandoos;

II. Em todo o ciclo de vida e contexto de empreendimentos, inclusive na sua gestão e manutenção; 
III. Na formação e atualização de futuros engenheiros e profissionais, envolvidos em projetos de produtos (bens e serviços) e empreendimentos.

As DCNs explicitam a importância do desenvolvimento do perfil empreendedor do engenheiro, tanto na concepção de soluções de engenharia, como na gestão do processo de produção dessas soluções, além da adequação do perfil de formação à atividades de treinamento técnico e docência (BRASIL, 2019).

A configuração do currículo dos cursos de engenharia, em uma perspectiva de desenvolvimento de competências, deve necessariamente possibilitar situações em que os estudantes vivenciem situações de resolução de problemas de sua prática profissional. Ao longo do curso, o nível de dificuldade e envolvimento em tais atividades deve aumentar, conforme o desenvolvimento das competências nos alunos (BRASIL, 2019).

\subsection{Projeto pedagógico do curso}

Uma vez concebido o perfil do egresso, cabe ao PPC estruturar as vias para a concretização do mesmo. Nessa perspectiva, conforme Brasil (2019), o projeto pedagógico deve trazer "[...] as diferentes iniciativas do processo de formação e sua forma de articulação para atingir os resultados esperados $[\ldots]$...

Torna-se importante a análise do papel da instituição de ensino no contexto local, bem como as diretrizes do plano de desenvolvimento institucional, visão, missão e valores institucionais, alinhados com as particularidades do curso de engenharia (BRASIL, 2019).

No que tange às metodologias de ensino, destaca-se a importância de oportunizar o desenvolvimento da autonomia dos alunos, que "[...] de forma pluridimensional, que leve em consideração os pilares do conhecimento: aprender a conhecer, aprender a fazer, aprender a conviver e aprender a ser." (BRASIL, 2019). Tais pilares amparam a atuação profissional do egresso, a fim de promover, através da engenharia, transformações sociais através do desenvolvimento científico e tecnológico.

De acordo com Bacich e Moran (2018), as metodologias ativas priorizam o papel protagonista do aluno, assumindo uma postura interativa no processo de ensino e aprendizagem. Assim, exige-se que o aluno não apenas ouça, mas desenvolva atividades de ordem superior, com oportunidade de refletir sobre o que estão desenvolvendo (BONWELL e EISON, 1991).

Para que isso seja possível, é necessário algumas quebras de paradigmas nas atividades acadêmicas. No projeto pedagógico do curso deve estar claro como as metodologias ativas serão articuladas, conforme CN-DCNs (2020) recomenda-se que:

Os PPCs estabeleçam claramente como serão aplicadas as metodologias ativas no processo de aprendizagem.

Os estudantes exerçam papel ativo, tornando-se protagonistas do processo de aprendizagem.

Sejam estimuladas metodologias que visam desenvolver no estudante a capacidade de resolver problemas usando conhecimento multidisciplinar. $\mathrm{O}$ estudo baseado na problematização deve privilegiar atividades que incentivem o estudo individual e em grupo, o ensino tutorial centrado no estudante, o manejo de bancos de dados, o acesso a fontes bibliográficas e aos recursos de informática e outras técnicas pedagógicas.

Estejam previstas atividades interdisciplinares e transdisciplinares (projetos de final de curso, estágios, projetos integrados, projetos de extensão, entre outros).

O PPC deve estimular, dentre as diversas metodologias ativas disponíveis, aquelas que ativam e/ou que desenvolvem o pensamento crítico no processo de aprendizagem. 
No contexto da utilização de metodologias de ensino ativas, destaca-se, no processo de avaliação que

[...] devem ser estimuladas as atividades acadêmicas, tais como trabalhos de iniciação científica, projetos interdisciplinares e transdisciplinares, projetos de extensão, visitas técnicas, trabalhos em equipe, desenvolvimento de protótipos, monitorias, participação em empresas juniores e outras atividades empreendedoras. (BRASIL, 2019, p. 28).

Nessa perspectiva, as metodologias ativas contribuem para que, ao final do curso, o egresso esteja preparado para enfrentar os desafios profissionais atrelados à área tecnológica, que são dinâmicos e exigem do profissional alta capacidade de pesquisar, aprender e agir.

Conforme Brasil (2019), as diretrizes devem estar contempladas no perfil do egresso, cabendo ao PPC oportunizar meios de desenvolvimento das competências, cumprindo assim os objetivos do cursos. Assim, necessita-se estabelecer os conteúdos e a forma como serão trabalhados, e ao final, devem ser sintetizados no projeto de final de curso.

As DCNs publicadas em 2019 ratificam a necessidade da formação a partir de uma sólida base comum, e com o avanço dos estudos, haja opção de aprofundamento em uma ou mais ênfases para determinados campos de atuação, assim vem à tona algumas recomendações das DCNs apresentadas conforme Brasil (1997, apud BRASIL, 2019, p. 29), dando ênfase ao:

[...] (i) o estímulo a uma sólida formação geral, necessária para que o futuro graduado possa vir a superar os desafios de renovadas condições de exercício profissional e de produção do conhecimento; e,

(ii) ampla liberdade na composição da carga horária a ser cumprida para a integralização dos currículos, assim como na especificação das unidades de estudos a serem ministradas; entre outros aspectos.

A proposição das diretrizes são amparadas por seis tópicos fundamentais: foco na formação através do desenvolvimento das competências, metodologias inovadoras, indução de políticas institucionais inovadoras, ênfase na gestão do processo de aprendizagem, fortalecimento do relacionamento com diferentes organizações e valorização da formação do corpo docente BRASIL (2019).

No que tange às competências, o Engenheiro ou Engenheira deve ser capaz de observar, formular e resolver problemas a partir da aplicação da tecnologia. Essa premissa traz à tona a necessidade da formação ser vista como um processo com foco nas pessoas envolvidas. $\mathrm{Ou}$ seja, não cabe a visão que a formação em Engenharia é composta por um conjunto de conhecimentos que o aluno adquire a cada período durante o curso BRASIL (2019).

As soluções tecnológicas oferecidas pelo egresso serão compostas, conforme Brasil (2019, p. 29), pelo "[...] conhecimento técnico da matemática, das ciências, das ciências da engenharia, para que se alcance o resultado que seja tecnicamente viável e desejável para o usuário final". Para isso, além do conhecimento técnico, é imprescindível o senso humano e habilidades empreendedoras, para que as soluções oferecidas supram às necessidades do usuário, sejam economicamente viáveis e ambientalmente adequadas (BRASIL, 2019).

Quanto às metodologias inovadoras, há a necessidade de ruptura de um modelo tradicional, com foco na transmissão de conhecimento, para um processo ensino aprendizagem em que o aluno seja protagonista. Dessa forma, conforme Brasil (2019, p. 30), "[...] ganham destaque as metodologias tal como o ensino baseado em projetos, ou Project Based Learning (PBL), com lastro no desenvolvimento de competências, na aprendizagem colaborativa e na interdisciplinaridade". 
A intenção da utilização de tais metodologias consiste em engajar os alunos no processo de ensino e aprendizagem desde o início do curso através do seu protagonismo, aperfeiçoando tópicos de qualidade de ensino e diminuindo a evasão escolar (BRASIL, 2019).

No que tange às políticas institucionais inovadoras, destaca-se a necessidade da flexibilidade das DCNs para adequação ao contexto da instituição de ensino, resultando em um projeto pedagógico com coerência entre as diretrizes e o papel institucional perante à sociedade (BRASIL, 2019).

A ênfase na gestão do processo de aprendizagem atua na análise do cumprimento dos objetivos do curso, na formação das competências e a sua adequação à sociedade, que possibilita a intervenção efetiva do profissional. Assim, com a constatação de eventuais necessidades alterações, possa ser tomadas as devidas conclusões (BRASIL, 2019).

As DCNs buscam o fortalecimento do relacionamento com outras instituições pelo fato de possibilitar a contextualização do ensino no ambiente de atuação do engenheiro. Assim, deve ser desenvolvidos atividades, tais como desenvolvimento de projetos, integração de docentes com profissionais das empresas, tanto nas atividades do curso como nas atividades empresariais, projetos de final de curso voltados a problemas reais, além das oportunidades de estágio. A interação entre instituição de ensino e empresas privadas e públicas, há a necessidade de uma relação do curso superior com a sociedade em geral, que pode ser efetivada através da extensão (BRASIL, 2019).

No que trata a carga horária e o tempo de integralização do curso, deve-se levar em conta a grande defasagem de aprendizagem dos ingressantes nos cursos de engenharia, assim uma parcela da carga horária do curso de engenharia é dedicada a retomada de conteúdos básicos, necessários à carreira da engenharia, afim de contornar alguns problemas na educação básica. Diante disso, conforme Brasil (2019, p. 32):

[...] recomenda-se a manutenção, como tempo referencial, da legislação em vigor, a fim de garantir a adaptação adequada dos ingressantes no ensino superior, bem como o tempo necessário para a maturação dos estudantes e da formação específica, que se alinhe com as necessidades do mercado e da sociedade e, desse modo, garantir a empregabilidade dos egressos ou seu êxito como empreendedores.

A estrutura curricular deve conter os conteúdos básicos, profissionais e específicos de acordo com a ênfase da formação, e devem ser trabalhados de forma contextualizada. Conforme necessidades específicas, as instituições de ensino podem incluir conteúdos que atendam também, demandas locais (BRASIL, 2019).

Devido às mudanças impostas pelas mudanças tecnológicas no cenário profissional, a demanda de mão de obra pode ser alterada, e é imprescindível aos profissionais da engenharia a capacidade de adequação a essas mudanças, acarretando a necessidade de novas competências e habilidades específicas (BRASIL, 2019).

O estágio é um importante instrumento que possibilita a prática profissional. O estágio obrigatório, com carga horária mínima de 160 horas, deve ser realizado em organizações que desenvolvem atividades de engenharia, possibilitando a interação entre profissionais da área, docentes e discentes (BRASIL, 2019).

O projeto de final de curso, deve ter uma regulamentação própria, e tem um papel fundamental na síntese do curso. Dessa forma, há um campo com imensas possibilidades interdisciplinares com atuação focada na comunidade. Ainda conforme Brasil (2019) "Considerando as inovações assumidas no processo de aprendizagem, cabe reconhecer a possibilidade de diversificação de experiências na consecução desse objetivo e da sua forma de apresentação". 
Como forma de enriquecer a formação do estudante de engenharia, as atividades complementares constituem um componente curricular. Devem ser priorizadas atividades fora do ambiente da instituição, com diversidade de conhecimentos e interesses. Brasil (2019).

A inserção do curso de Engenharia na sociedade é muito importante. Dessa forma, segundo Brasil (2019, p. 34) "As atividades de extensão estão contempladas nas Diretrizes Curriculares Nacionais do Curso de Engenharia como componente da organização curricular, obedecendo às normas pertinentes, expedidas no âmbito do Conselho Nacional de Educação (CNE)".

\section{AÇÕES REALIZADAS NO CURSO DE ENGENHARIA MECÂNICA DO IFRS CAMPUS IBIRUBÁ}

O curso de Engenharia Mecânica do IFRS Campus Ibirubá, teve sua primeira turma ofertada no ano de 2015 e surgiu para suprir a demanda regional por profissionais qualificados na área de Engenharia Mecânica. O curso também oportuniza a continuidade dos estudos dos egressos dos cursos técnicos da instituição (IFRS, 2017).

De acordo com IFRS (2017, p. 25):

A concepção do curso se orienta pela perspectiva de formação generalista, humanística, crítica e reflexiva, visando a formação de um profissional capaz de absorver e desenvolver novas tecnologias, estimulando a sua atuação crítica e criativa na identificação e resolução de problemas. Para tanto a proposta pedagógica do curso entende que o processo de formação do educando, deve integrar teoria e prática, buscando uma formação que possa promover transformações significativas para o desenvolvimento social.

Na perspectiva de uma formação generalista com um olhar voltado às demandas locais, o PPC apresenta como objetivos a formação de profissionais com competências e habilidade conforme a Resolução CNE/CES, de 11 de março de 2002, apresentada em BRASIL (2002 apud IFRS, 2017, p. 21).

Quanto aos procedimentos metodológicos, o programa do curso fomenta a consolidação de situações de ensino com características didáticas tais como: coleta de informações, investigação e pesquisa, fixação e contextualização, cooperação, avaliação significativa da aprendizagem. A diversificação dos espaços de aprendizagem, tais como: seminários integradores, trabalhos campo, visitas técnicas, eventos científicos, trabalhos em equipe, práticas em laboratório, grupos de pesquisa, grupos de monitoria, palestras, semanas acadêmicas, integração com os demais cursos da instituição, colaboram na consolidação de momentos significativos de ensino aprendizagem (IFRS, 2017).

As ações interdisciplinares são desenvolvidas através de projetos integradores, desenvolvidos nos componentes curriculares de Gerenciamento de Projetos e Projeto Mecânico, ofertados no oitavo e nono semestre, respectivamente. Além disso, o currículo está estruturado de tal forma que diferentes áreas do conhecimento se cruzam nos próprios componentes, com a possibilidade de dois ou mais docentes atuarem de forma colegiada em um mesmo componente curricular (IFRS, 2017).

Como exemplo do que já vem sendo executado, tem-se no Quadro 1, os projetos integradores realizados nos anos de 2018 e 2019, que foram apresentados em eventos científicos da instituição. 
Quadro 1 - Projetos integradores realizados nos anos de 2018 e 2019

\begin{tabular}{|l|c|}
\hline \multicolumn{1}{|c|}{ Título do Trabalho } & Referência \\
\hline $\begin{array}{l}\text { Projeto de dobradeira de tubos hidráulica com acionamento } \\
\text { manual }\end{array}$ & Cancian et al. (2019) \\
\hline Dispositivo para dobrar chapas & Fredrich et al. (2019) \\
\hline Projeto e construção do protótipo de uma calandra & Pereira et al. (2019) \\
\hline $\begin{array}{l}\text { Protótipo de um robô seguidor de linha para limpeza de pisos } \\
\text { industriais }\end{array}$ & Schneider et al. (2019) \\
\hline $\begin{array}{l}\text { Projeto informacional de uma barra pulverizadora articulada } \\
\text { Projeto informacional para o desenvolvimento de um veículo } \\
\text { elétrico de carga }\end{array}$ & Oliveira et al. (2018) \\
\hline $\begin{array}{l}\text { Projeto informacional de um dispositivo de montagem para } \\
\text { linhas de semente de uma semeadora de inverno }\end{array}$ & Meinke et al. (2018) \\
\hline $\begin{array}{l}\text { Projeto informacional para desenvolvimento de um picador } \\
\text { de soqueiras }\end{array}$ & Schneider et al. (2018) \\
\hline $\begin{array}{l}\text { Projeto informacional de um dispositivo para o transporte de } \\
\text { uma ensiladeira }\end{array}$ & Lagemann et al. (2018) \\
\hline $\begin{array}{l}\text { Projeto informacional de um aplicativo para distribuidor de } \\
\text { sementes }\end{array}$ & Amaral et al. (2018) \\
\hline $\begin{array}{l}\text { Projeto informacional de um guincho de utilização agrícola } \\
\text { Projeto informacional de uma turbina a vapor de pequeno } \\
\text { porte para fins didáticos }\end{array}$ & Reckziegel et al. (2018) \\
\hline $\begin{array}{l}\text { Projeto informacional de um dispositivo para monitoramento } \\
\text { do ruído em automóveis }\end{array}$ & Marcena et al. (2018) \\
\hline $\begin{array}{l}\text { Projeto informacional de um sistema de enlonamento } \\
\text { automático para veículos de carga }\end{array}$ & Doll et als) \\
\hline
\end{tabular}

Fonte: Autor

No ano de 2019, ações de ensino, articulada à pesquisa e extensão, resultaram na apresentação de trabalhos, tais como os trabalhos de Ebert et al. (2019), Santos et al. (2019), Paulus et al. (2019), Silva et al. (2019), Beskow et al. (2019) e Berwig et al. (2019), o que incentiva a iniciação à publicação estudantes.

Além de tais atividades já realizadas, há um grande campo a ser explorado, quando da análise do PPC para adequação às novas DCNs, como a ampliação dos projetos integradores, articulados a projetos de ensino, pesquisa e extensão, com foco na resolução de problemas da sociedade, além da sistematização da participação dos alunos em eventos científicos. Esses pontos levantados são demandas que necessitam serem contempladas no PPC do curso com vistas a adequação às novas DCNs. 


\section{CONSIDERAÇÕES FINAIS}

Este trabalho trouxe um referencial sobre as novas DCNs, onde destaca-se a importância de traçar o perfil do egresso, bem como a forma de desenvolvimento desse perfil nos alunos. As DCNs partem da primazia de utilização de metodologias ativas nos diversos ambientes e situações de ensino, para que o discente seja protagonista de sua aprendizagem, e tenha condições de atuar ativamente frente aos desafios profissionais de sua carreira.

No curso de Engenharia Mecânica do IFRS Campus Ibirubá, as metodologias ativas já são empregadas nos componentes curriculares de Gerenciamento de Projeto e Projeto Mecânico, de forma formalizada através do atual PPC. Além disso, atividades integradoras também são realizadas em outros componentes, partindo da iniciativa dos docentes pela utilização de metodologias inovadores em sala de aula. Conforme apresentado, incentiva-se a participação dos alunos em eventos científicos institucionais. Tais atividades vêm ao encontro das novas DCNs. Porém, é necessário a devida sistematização e formalização de tais práticas no PPC para a efetiva adequação às novas DCNs.

É um grande desafio a adequação dos cursos de graduação em Engenharia às novas DCNs, mas o processo reflexivo sobre o curso contribui para identificar o que está sendo realizado de positivo, e as oportunidades de aprimoramento e recondução dos pontos deficitários.

\section{REFERÊNCIAS}

AMARAL, W.; CELLA, M; BAAL, E. Projeto informacional de um aplicativo para distribuidor de sementes. In: VII Moepex: Mostra de ensino pesquisa e extensão, 2018, Bento Gonçalves. Anais. Ibirubá, 2018.

BACICH, Lilian; MORAN, José (orgs.). Metodologias ativas para uma educação inovadora: uma abordagem teórico-prática. 1ª ed. Porto Alegre: Editora Penso, 2018.

BERVIG, Igor R. dos S.; SCHLEDER, Giancarlo S.; ANDARA Flávio R. Projeto, desenvolvimento e construção de uma afiadora de ferramentas de usinagem. In: XVII Mostra de Ensino, Pesquisa e Extensão, 2019, Bento Gonçalves. Anais. Ibirubá, 2019.

BESKOW, Stéfany; SILVEIRA,Vitor H. M. da; ANDARA Flávio R. Processo Shaw: uma alternativa tecnológica ao processo de fundição em areia convencional. In: XVII Mostra de Ensino, Pesquisa e Extensão, 2019, Bento Gonçalves. Anais. Ibirubá, 2019.

BONWELL, C. C.; EISON, J. A. Active learning: creating excitement in the classroom. 1991. Disponível em: https:// www.ericdigests.org/ 1992-4/ active.htm . Acesso em: 10 maio 2020.

BRASIL. Ministério da Educação. Conselho Nacional de Educação. Câmara de educação Superior. Resolução no 2, de 24 de abril de 2019. Institui as Diretrizes Curriculares Nacionais do Curso de Graduação em Engenharia. Brasília, DF: Ministério da Educação, 2019. Disponível em:

http://portal.mec.gov.br/index.php?option=com_docman\&view=download\&alias=112681rces002-19\&category_slug=abril-2019-pdf\&Itemid=30192. Acesso em: 28 maio 2020. 
COMISSÃO NACIONAL PARA IMPLANTAÇÃO DAS NOVAS DIRETRIZES CURRICULARES NACIONAIS DO CURSO DE GRADUAÇÃO EM ENGENHARIA (CNDCNs). Relatório síntese. [S.1.]: [s.n], 2020.

COSTA, L. O.; NASCIMENTO, R. L.; E.. Projeto informacional de um dispositivo para monitoramento do ruído em automóveis. In: VII Moepex: Mostra de ensino pesquisa e extensão, 2018, Bento Gonçalves. Anais. Ibirubá, 2018.

DOLL, F. P.; NEGRELLO, G.; SANTOS, M. P.; BAAL, E. . Projeto informacional de um sistema de enlonamento automático para veículos de carga. In: VII Moepex: Mostra de ensino pesquisa e extensão, 2018, Bento Gonçalves. Anais. Ibirubá, 2018.

EBERT, Ana C.; SILVEIRA,Vitor H. M. da; ANDARA Flávio R. Fundição de monocristais: uma revisão. In: XVII Mostra de Ensino, Pesquisa e Extensão, 2019, Bento Gonçalves.

Anais. Ibirubá, 2019.

INSTITUTO FEDERAL DE EDUCAÇÃO, CIÊNCIA E TECNOLOGIA DO RIO GRANDE DO SUL. Projeto pedagógico do cursos de engenharia mecânica. Ibirubá: IFRS, 2017.

LAGEMANN, M. B.; SILVA, V. C. M.; BAAL, E. . Projeto informacional de um dispositivo para o transporte de uma ensiladeira. In: VII Moepex: Mostra de Ensino Pesquisa e Extensão, 2018, Bento Gonçalves. Anais. Ibirubá, 2018.

MARCENA, Y. K.; DALCIN, P. A.; BAAL, E. . Projeto informacional de uma turbina a vapor de pequeno porte para fins didáticos. In: VII Moepex: Mostra de ensino pesquisa e extensão, 2018, Bento Gonçalves. Anais. Ibirubá, 2018.

MEINKE, J. V.; MARTINS, P. F.; HiLGERT, R; BAAL, E. . Projeto informacional de um dispositivo de montagem para linhas de semente de uma semeadora de inverno. In: VII Moepex: Mostra de Ensino pesquisa e extensão, 2018, Bento Gonçalves. Anais. Ibirubá, 2018.

MELLO, F.; ORLANDI, G. T.; FRANCO, B. C.; RATHKE, J. E.; BAAL, E. . Projeto informacional para o desenvolvimento de um veículo elétrico de carga. In: VII Moepex: Mostra de Ensino Pesquisa e Extensão, 2018, Bento Gonçalves. Anais. Ibirubá, 2018.

\section{MOBILIZAÇÃO EMPRESARIAL PELA INOVAÇÃO. Documento de apoio à implantação das DCNs do curso de graduação em engenharia. Brasília: CNI, 2020.}

OLIVEIRA, E. J.; HENKES, F. P.; SOUZA, S. G.; BAAL, E. . Projeto informacional de uma barra pulverizadora articulada. In: VII Moepex - Mostra de Ensino Pesquisa e Extensão, 2018, Bento Gonçalves. Anais. Ibirubá, 2018.

PAULUS, Rafael B.; ANDARA Flávio R.; SILVEIRA,Vitor H. M. da. Avaliação da temperabilidade de aços com $0,4 \%$ de carbono e diferentes teores de elementos de liga sujeitos a condições de ensaio no inverno. In: XVII Mostra de Ensino, Pesquisa e Extensão, 2019, Bento Gonçalves. Anais. Ibirubá, 2019. 
RECKZIEGEL, R.; HOLZ, V. H.; FREDRICH, M.; BAAL, E. . Projeto informacional de um guincho de utilização agrícola. In: VII Moepex: Mostra de ensino pesquisa e extensão, 2018, Bento Gonçalves. Anais. Ibirubá, 2018.

SANTOS, Álisson A.; ANDARA Flávio R.; SILVEIRA, Vitor H. M. da. Solidificação unidirecional: fundição com foco em microestrutura. In: XVII Mostra de Ensino, Pesquisa e Extensão, 2019, Bento Gonçalves. Anais. Ibirubá, 2019.

SCHNEIDER, C. J.; CANCIAN, A. L. H.; DALMOLIN, I. I.; BAAL, E. . Projeto informacional para desenvolvimento de um picador de soqueiras. In: VII Moepex: Mostra de Ensino Pesquisa e Extensão, 2018, Ibirubá. Apresentações e Autores, 2018.

SCHWANTES, N. D.; WELTER, W.; BAAL, E. . Projeto informacional para o desenvolvimento de um plano de manutenção preventiva. In: VII Moepex: Mostra de ensino pesquisa e extensão, 2018, Bento Gonçalves. Anais. Ibirubá, 2018.

SILVA, Igor R. da; ANDARA Flávio R.; SILVEIRA,Vitor H. M. da. Squeeze Casting: um processo híbrido entre Fundição e Extrusão. In: XVII Mostra de Ensino, Pesquisa e Extensão, 2019, Bento Gonçalves. Anais. Ibirubá, 2019.

\title{
ANALYSIS OF THE NEW NATIONAL CURRICULAR GUIDELINES WITH APPLICATION IN A MECHANICAL ENGINEERING COURSE
}

\begin{abstract}
This paper presents a review of the new National Curricular Guidelines for the Undergraduate Engineering Course (DCNs) and presents actions in accordance with these guidelines and which are already carried out within the scope of the Mechanical Engineering course of the Federal Institute of Education, Science and Technology of Rio Grande do Sul (IFRS) Campus Ibirubá. The new DCNs were published in 2019, in order to improve the quality of undergraduate engineering courses in the country, considering the demands of the sectors of society where Engineers work. The adequacy to the guidelines leads to an important reflection on the pedagogical project of the course, being evidenced in this process positive and negative points of the undergraduate course. In the revision of the document of the new DCNs, emphasis is placed on encouraging the use of active methodologies and encouraging students' scientific initiation. The search for some actions already carried out within the scope of the course occurred through the search for works published by students in institutional scientific events. Twenty papers were listed that demonstrate points in common between the course and what excites the new DCNs, with regard to the active methodologies and the encouragement of students' scientific initiation. Still, there is a need to systematize and formalize such actions in the pedagogical project of the course for the proper adaptation to the new DCNs.
\end{abstract}

Keywords: National curricular guidelines. Mechanical Engineering. Active methodologies. 